\title{
Obesity, insulin resistance, adipocytokines and breast cancer: New biomarkers and attractive therapeutic targets
}

\author{
Maria Dalamaga
}

Maria Dalamaga, Department of Clinical Biochemistry, Medical School, University of Athens, "Attikon" General University Hospital, 12462 Athens, Greece

Author contributions: Dalamaga M solely contributed to this paper.

Correspondence to: Maria Dalamaga, MD, PhD, MS, MPH, Assistant Professor, Department of Clinical Biochemistry, Medical School, University of Athens, "Attikon" General University Hospital, 1 Rimini Street, Chaidari, 12462 Athens,

Greece.madalamaga@med.uoa.gr

Telephone: $+30-210-5831915$ Fax: $+30-210-6082467$

Received: May 26, $2013 \quad$ Revised: July 8, 2013

Accepted: August 16, 2013

Published online: August 20, 2013

\section{Abstract}

Worldwide, breast cancer $(B C)$ represents the most common type of non-skin human malignancy and the second leading cause of cancer-related deaths amid women in Western countries. Obesity and its metabolic complications have rapidly become major global health issues and are associated with increased risk for cancer, especially BC in postmenopausal women. Adipose tissue is considered as a genuine endocrine organ secreting a variety of bioactive adipokines, such as leptin, adiponectin, resistin and nicotinamide phosphoribosyltransferase/visfatin. Recent evidence has indicated that the constellation of obesity, insulin resistance and adipokines is associated with the risk and prognosis of postmenopausal BC. Direct evidence is growing rapidly supporting the stimulating and/or inhibiting role of adipokines in the process of development and progression of BC. Adipokines could exert their effects on the normal and neoplastic mammary tissue by endocrine, paracrine and autocrine mechanisms. Recent studies support a role of adipokines as novel risk factors and potential diagnostic and prognostic biomarkers in BC. This editorial aims at providing important insights into the potential pathophysiological mechanisms linking adipokines to the etiopathogenesis of $\mathrm{BC}$ in the context of a dysfunctional adipose tissue and insulin resistance in obesity. A better understanding of these mechanisms may be important for the development of attractive preventive and therapeutic strategies against obesity-related breast malignancy.

\section{(c) 2013 Baishideng. All rights reserved.}

Key words: Breast cancer; Obesity; Insulin resistance; Adipokines; Adiponectin; Resistin; Leptin; Nicotinamide phosphoribosyl-transferase; Visfatin

Core tip: Recent evidence has shown that the constellation of obesity, insulin resistance and adipokines is associated with the risk and prognosis of postmenopausal breast cancer $(\mathrm{BC})$. Direct evidence is growing rapidly supporting the stimulating and/or inhibiting role of adipokines in the process of development and progression of BC. Recent studies support a role of adipokines as novel risk factors and potential diagnostic and prognostic biomarkers in BC. This editorial aims at providing important insight into the potential pathophysiological mechanisms linking adipokines to the etiopathogenesis of $\mathrm{BC}$ in the context of a dysfunctional adipose tissue and insulin resistance in obesity. Understanding of these mechanisms may be important for the development of attractive preventive and therapeutic strategies against obesity-related breast malignancy.

Dalamaga M. Obesity, insulin resistance, adipocytokines and breast cancer: New biomarkers and attractive therapeutic targets. World $J$ Exp Med 2013; 3(3): 34-42 Available from: URL: http://www. wjgnet.com/2220-315X/full/v3/i3/34.htm DOI: http://dx.doi. org/10.5493/wjem.v3.i3.34

\section{INTRODUCTION}

Worldwide, breast cancer (BC) represents the most common type of non-skin human malignancy and the second lead- 
ing cause of cancer-related deaths amid women in Western countries $^{[1,2]}$. The prevalence of BC increases with age and, therefore, $\mathrm{BC}$ is more common in postmenopausal than premenopausal women. Despite substantial progress in BC treatment, metastatic disease, occurring in $50 \%$ of patients following radical surgery, remains incurable $\mathrm{e}^{[3-7]}$.

Apart from risk factors such as germline mutation in BRCA1, family history of BC or diagnosed carcinoma in situ, common well-established risk factors for BC, particularly postmenopausal BC (PBC), are hormone-associated reproductive factors such as earlier age at menarche, later age at menopause, older age at first birth, decreased parity and use of hormone therapy (HT); anthropometric features such as increased final height, weight, body mass index (BMI), waist circumference; atypical hyperplasia of the mammary gland; and high breast density on mammographic screening ${ }^{[4-6]}$. Alcohol consumption is considered a modest risk for BC risk, possibly by enhancing estrogen levels ${ }^{[4-9]}$. Nevertheless, more than $50 \%$ of BCs arise in the absence of known common risk factors $^{[4-9]}$.

\section{OBESITY, INSULIN RESISTANCE}

\section{AND BC}

A meta-analysis and systematic review in conjunction with other evidence have linked obesity to excess risk for many cancers, including $\mathrm{BC}^{[1-3]}$. Obesity represents a growing global public health issue in industrialized countries affecting a significant part of the population across all age, gender and ethnic groups ${ }^{[2]}$. There is accumulating evidence that overweight/obesity constitutes a risk factor for BC in postmenopausal women ${ }^{[2-5]}$. The excess of body weight significantly increases PBC risk by $30 \%-50 \%{ }^{[10]}$. However, based on epidemiological data, obesity has been associated with decreased or neutral $\mathrm{BC}$ risk in premenopausal women ${ }^{[6,10,11]}$. Whilst increased birthweight is associated with premenopausal BC, weight gain acquired later in life, after the age of 40 and mainly during the perimenopausal period, presents the most deleterious effects ${ }^{\left[{ }^{[]}\right.}$. The effect of the BMI increase on BC risk is particularly observed in tumors with positive estrogen (ER) and progesterone receptor (PR) tumors and in HT non-users ${ }^{\left[{ }^{[]}\right.}$. Obesity is also associated with increased tumor burden and histopathological grade, and a higher incidence of lymph node metastasis in BC patients. In addition to an increased risk of developing BC, overweight/ obese and physically inactive patients appear to be at increased risk for BC progression and BC-related mortality regardless of menopausal status ${ }^{[1-3,9]}$.

The mechanism connecting obesity with $\mathrm{PBC}$ is not completely elucidated. After menopause, adipose tissue is the main site of peripheral aromatization of androgens to estrogens, which may induce mitogenic activity in mammary epithelial cells ${ }^{[3,12]}$. Excess adiposity is associated with elevated estrogen levels. Obese postmenopausal women with $\mathrm{BC}$ present significantly higher total and free estradiol levels as well as increased local estro- gen levels within breast tumors compared to healthy women $^{[6,7]}$.

Additionally, increased adiposity and, in particular increased visceral fat may cause hyperinsulinemia, insulin resistance and dyslipidemia. In turn, hyperinsulinemia leads to higher insulin-like growth factor-I (IGF-I) levels exerting a mitogenic effect on both normal and neoplastic breast epithelial cell as well as lowers the hepatic synthesis of sex hormone binding globulin resulting in an increase of the bioavailable fraction of both estradiol and testosterone ${ }^{[3,12]}$. Epidemiological evidence has indicated that both pre- and postmenopausal women with insulin resistance, metabolic syndrome and type 2 diabetes $(\mathrm{t} 2 \mathrm{DM})$ have an increased $\mathrm{BC}$ risk $^{[3,13]}$. According to the International Diabetes Federation, the metabolic syndrome is defined as a cluster of conditions that include central (abdominal) obesity based on ethnicity specific values for waist circumference associated with any two of the following four factors: (1) hypertriglyceridemi a $[\geqslant 150 \mathrm{mg} / \mathrm{dL}(\geqslant 1.7 \mathrm{mmol} / \mathrm{L})$ or specific treatment for this lipid abnormality]; (2) reduced HDL cholesterol $[<40 \mathrm{mg} / \mathrm{dL}(<1.03 \mathrm{mmol} / \mathrm{L})$ in males, $<50 \mathrm{mg} / \mathrm{dL}(<$ $1.29 \mathrm{mmol} / \mathrm{L})$ in females, or specific treatment for this lipid abnormality]; (3) hypertension (systolic blood pressure $\geqslant 130$ or diastolic blood pressure $\geqslant 85 \mathrm{mmHg}$ or treatment of previously diagnosed hypertension); and (4) raised fasting plasma glucose levels $[\geqslant 100 \mathrm{mg} / \mathrm{dL}(\geqslant$ $5.6 \mathrm{mmol} / \mathrm{L})$ or previously diagnosed type 2 diabetes $]^{[14]}$. The metabolic syndrome is associated with increased risk of t2DM and cardiovascular disease ${ }^{[14]}$.

The insulin-IGF-I pathway may lead to the activation of various intracellular pathways, including mitogenactivated protein kinase (MAPK) and phosphatidylinositol 3-kinase (PI3K) signaling cascade affecting tumor growth ${ }^{[3,12]}$. Moreover, estrogen and the insulin-IGF-I pathways intersect at the $\mathrm{G}_{1}-\mathrm{S}$ phase of cell-cycle progression and synergistically induce mitogenic effects on breast epithelium. The insulin-IGF-I pathway may activate ER- $\alpha$ transcriptional activity in BC cell lines even in the absence of estradiol ${ }^{[6,12]}$.

Visceral adipose tissue plays a pivotal role in the development of a systemic inflammatory state contributing to obesity-related metabolic diseases ${ }^{[2,3]}$. Excess body weight is considered a subclinical chronic low-grade inflammatory and prothrombotic state involved in obesity-associated insulin resistance and cancer ${ }^{[2,3]}$. The activation of proinflammatory adipocytokines and the suppression of anti-inflammatory adipocytokines such as adiponectin increase the hepatic synthesis of acute phase reactants, establishing therefore a positive feed-back loop and enhancing the systemic inflammatory state which promotes cancerinogenesis ${ }^{[3]}$. At the same time, lipid accumulation increases demand on the endoplasmic reticulum resulting in an uncontrolled production of reactive oxygen species (ROS) which stimulate inflammatory signaling pathways and induce endoplasmic reticular stress, oxidative stress and DNA damage leading to genomic instability ${ }^{[15]}$. It is well known that oxidative stress which reflects an 
imbalance between the systemic manifestation of ROS and the biological system's ability to detoxify the reactive intermediates or to repair the resulting damage, may cause toxic effects through the production of peroxides and free radicals that damage all components of the cell, including $\mathrm{DNA}^{[15]}$. Moreover, oxidative stress may cause disruptions in normal mechanisms of cellular signaling. As the adipose tissue expands in obesity, the vasculature is not sufficient to oxygenate adequately the adipocytes leading to hypoxia. The resultant hypoxia, mediated by the hypoxia-inducible factor- I, in conjunction with endoplasmic reticular stress and oxidative stress initiate a pro-inflammatory cascade with overproduction of tumor necrosis factor- $\alpha$ (TNF- $\alpha$ ) and interleukin (IL)-6, through nuclear transcription factor- $\kappa \mathrm{B}(\mathrm{NF}-\kappa \mathrm{B})$ activation, stimulating the systemic inflammatory state which further promotes tumor growth ${ }^{[3]}$.

\section{ADIPOSE TISSUE, ADIPOKINES AND BC}

Apart from its lipid storage function, adipose tissue constitutes an active endocrine organ secreting several bioactive adipocytokines or adipokines as well as inflammatory cytokines, regulating physiological and pathological processes, such as appetite, insulin sensitivity and resistance, inflammation, immunity, hematopoiesis and angiogenesis ${ }^{[3]}$. The mechanisms connecting excess adiposity in overweight/obesity with molecular and cellular pathways critical for cancerinogenesis involve innate and acquired immune activation, exposure to protumorigenic adipokines and growth factors as well as increased substrate availability to breast neoplastic cells. Adipocytes represent the majority of the breast tissue, with epithelial cells accounting for only $10 \%$ of breast volume $^{[7]}$. A recent hypothesis places adipocytes along with their autocrine, paracrine and endocrine functions at center stage in breast tumorigenesis ${ }^{[3,7]}$. The deregulated expression of adipokines may thus be involved in the association of obesity with BC. Although the exact interplay between adipokines is not yet well clarified, this editorial presents the role of main adipokines in breast carcinogenesis and examines the pathophysiological mechanisms that underlie the association between adipokines and breast malignancy in the context of a dysfunctional adipose tissue in obesity. Understanding of the mechanisms linking adipokines to $\mathrm{BC}$ is expected to be of importance in the development of preventive and therapeutic strategies.

\section{Leptin and BC}

Leptin, a 167-amino acid peptide that is primarily produced in adipose tissue, is a pleiotropic adipokine that regulates food intake, energy expenditure, immunity, inflammation, hematopoiesis, cell differentiation and proliferation ${ }^{[16,17]}$. Circulating leptin is directly proportional to the amount of body fat and fluctuates with acute changes in caloric intake, signaling the amount of energy stored in adipose tissue ${ }^{[17]}$. Common forms of obesity, insulin resistance and metabolic syndrome are associated with hyperleptinemia and leptin resistance ${ }^{[16]}$.

Leptin gene expression was found in normal breast epithelium, in BC cell lines as well as in solid tumors ${ }^{[7]}$. In the majority of cases with breast carcinoma, leptin was found to be overexpressed ${ }^{[7]}$. A growing body of evidence suggests that leptin exerts $\mathrm{BC}$ neoplastic effects via two mechanisms ${ }^{[16]}$. Firstly, leptin may act directly on BC cells by stimulating receptor-mediated signaling pathways leading to tumor cell growth, migration and invasion. Recently, in vitro studies have shown that leptin is involved in mammary tumorigenesis by stimulating tumor growth, cell survival and transformation, by amplifying ER $\alpha$ signaling that plays an critical role in hormone-dependent $\mathrm{BC}$ growth and progression and by upregulating the aromatase transcription which results in increased estrogen synthesis ${ }^{[18,19]}$. Leptin, through its receptor LepR, may promote growth and proliferation of BC cells via activation of various growth and survival signaling pathways including canonical: Janus Kinase 2/Signal Transducer and Activator of Transcription 3 (JAK2/STAT3), $\mathrm{PI} 3 \mathrm{~K} / \mathrm{v}$-Akt murine thymoma viral oncogene homolog/ mammalian target of rapamycin (PI3K/Akt/mTOR), mitogen-activated protein kinase/extracellular signalrelated kinase 1/2 (ERK1/2) and non-canonical signaling pathways such as protein kinase $\mathrm{C}$, c-Jun $\mathrm{N}$-terminal kinase $(\mathrm{JNK})$ and p38 MAPK ${ }^{[16,19,20]}$. Interestingly, this leptin activity is reinforced through entangled crosstalk with insulin, multiple oncogenes, cytokines and growth factors. For example, insulin via the PI3K and MAPK signaling pathways has induced leptin and LepR overexpression in human $\mathrm{BC}$ cells contributing to an autocrine stimulation of $\mathrm{BC}$ cell $^{[12]}$. Leptin has been shown in vitro to stimulate JNK in human BC cells in both a time- and a dose-dependent manner, with greater phosphorylated JNK levels after long-term exposure. JNK stimulation by leptin led to an upregulation of matrix metalloproteinase (MMP)-2 activity, which promotes cancer cell invasion ${ }^{[16,18-20]}$. It should be noted, however, that most in vitro studies have used extremely elevated leptin levels ${ }^{[16]}$. Secondly, leptin may act indirectly by decreasing tissue sensitivity to insulin causing hyperinsulinemia, by regulating inflammatory responses and shifting the $\mathrm{T}$ helper (TH) balance towards a TH1 phenotype with overproduction of cytokines such as IL-6, IL-12 and TNF- $\alpha$, and by influencing tumor angiogenesis; though such leptin effects were not seen in vivo ${ }^{[16]}$.

Nevertheless, in contrast to many in vitro studies, epidemiological studies have reported inconsistent and conflicting associations between circulating leptin levels and risk of $\mathrm{BC}^{[3,16]}$. Many studies have documented an association of hyperleptinemia with the risk for BC and advanced disease state ${ }^{[21]}$. In a recent prospective study, elevated prediagnostic leptin levels were associated with an increased risk of PBC independently from BMI ${ }^{[22]}$. However, other studies found no association of leptin levels with premenopausal or postmenopausal $\mathrm{BC}^{[7]}$. In 
addition, serum leptin levels did not appear to increase substantially the risk of pre-menopausal BC in situ and invasive pre- and post-menopausal $\mathrm{BC}^{[16,23]}$. So far, based on the available evidence, the utility of leptin as a $\mathrm{BC}$ biomarker is not clear. A possible association of $\mathrm{BC}$ with leptin needs to be analyzed further with larger prospective, longitudinal and mechanistic studies in order to prove causality and provide further insights into the paracrine and endocrine mechanisms underlying leptin's role in breast malignancy.

\section{Adiponectin and BC}

Adiponectin is a 244 -amino-acid, $30-\mathrm{kD}$ a protein secreted predominantly by white adipose tissue, sharing homology with collagen VIII, X, complement factor C1q, and tumor necrosis factor- $\alpha(\mathrm{TNF}-\alpha)^{[3]}$. Adiponectin exerts insulin-sensitizing, anti-inflammatory, anti-atherogenic, anti-neoplastic and cardioprotective effects as well as distinct effects on lipid metabolism ${ }^{[3,24]}$. Adiponectin may be found in different configurations presenting different biological effects: full-length, globular, low molecular weight, medium molecular weight and high molecular weight (HMW) adiponectin ${ }^{[3,24]}$. The HMW isoform represents the biologically active form of adiponectin, being strongly related with insulin resistance, metabolic syndrome and cardiovascular disease ${ }^{[3,24]}$. Adiponectin acts through its three receptors that have been identified; two main receptors: AdipoR1 and AdipoR2, and one receptor similar to the cadherin family ${ }^{[3,24]}$. Adiponectin stimulates several intracellular signaling pathways after binding to its receptors, mainly adenosine monophosphate (AMP)-activated protein kinase (AMPK), but also mTOR, NF- $\kappa$ B, JNK and STAT $3^{[3,24]}$.

Circulating adiponectin levels are generally determined in the range of 2 to $20 \mu \mathrm{g} / \mathrm{mL}^{[3,25]}$. Based on the assay methodology, race and gender, median adiponectin levels in healthy individuals with a BMI between 20 and $25 \mathrm{~kg} / \mathrm{m}^{2}$ are approximately $8 \mu \mathrm{g} / \mathrm{mL}$ for men and $12.5 \mu \mathrm{g} / \mathrm{mL}$ for women ${ }^{[25]}$. Hypoadiponectinemia is the common pathodenominator of the constellation of risk factors that synthesize the metabolic syndrome such as hypertension, dyslipidemia, obesity, hyperglycemia and insulin resistance ${ }^{[24]}$. Furthermore, in vivo hypoadiponectinemia has recently been found inversely associated with the risk of insulin resistance and obesity-associated malignancies, that is $\mathrm{BC}$, endometrial cancer, colon cancer, renal cancer, some hematologic malignancies of myeloid origin as well as gastric and prostate cancer ${ }^{[3]}$.

The majority of epidemiologic evidence has linked lower total or HMW adiponectin levels to an increased risk for BC independently of classical risk factors including leptin and the IGF-I system in both premenopausal and postmenopausal women ${ }^{[3,23,26]}$. Macis et a ${ }^{[27]}$ identified lower plasma circulating adiponectin levels in premenopausal women as a risk biomarker for progression from intraepithelial neoplasia to invasive cancer independently of age, BMI, and treatment group ${ }^{[3]}$. Adiponectin could play a role in $\mathrm{BC}$ etiopathogenesis, particularly in the low-estrogen environment observed in postmenopausal women $^{[3]}$. Because adipocytes constitute the predominant breast stromal element, adiponectin may exert a major paracrine and autocrine influence in mammary epithelium. Since AdipoR1/R2 are expressed in BC lines and tissues samples, adiponectin may act not only through altering the hormonal milieu but directly through inhibition of $\mathrm{BC}$ cells proliferation ${ }^{[28]}$. In addition, some but not all studies have pointed out that breast tumors arising in women with hypoadiponectinemia may present a more aggressive phenotype (higher histologic grade, large size of tumor and ER negativity $)^{[3]}$. Low adiponectin levels were associated with lymph node metastases and increased mortality in BC survivors after adjustment for parameters including obesity and insulin resistance ${ }^{[3]}$. Finally, some studies focusing on adiponectin genetic variants (ADIPOQ) and adiponectin receptor genes (ADIPOR1) and $\mathrm{BC}$ risk reported associations of ADIPOQ single nucleotide polymorphisms (SNPs) and ADIPOR1 SNP with BC risk ${ }^{[3]}$. However, other studies did not find such associations ${ }^{[3]}$.

Adiponectin exerts BC anti-neoplastic effects via two mechanisms: (1) it can act directly on BC cells by modulating receptor-mediated signaling pathways, including MAPK, AMPK, Wnt/ $\beta$-catenin and ER signaling; and (2) it can act indirectly by modulating insulin sensitivity at breast epithelium, influencing tumor angiogenesis and regulating inflammatory responses ${ }^{[3]}$. In vitro studies have indicated that adiponectin suppresses growth and promotes apoptosis of MCF-7 and MDA-MB-231 BC cell lines, and reduces the invasion of $\mathrm{BC}$ cells ${ }^{[3,29]}$. Adiponectin decreases also the secretion of proinflammatory cytokines (TNF- $\alpha$ and IL- 6 ) which are responsible for aromatase enhanced production in adipose tissue ${ }^{[3]}$. The role of adiponectin in tumor angiogenesis remains to be defined as both proangiogenic and anti-angiogenic activities toward mammary tumor growth have been described $^{[3]}$.

\section{Resistin and BC}

Resistin, also known as adipose tissue-specific secretory factor or found in inflammatory zone 3 , is a $12-\mathrm{kDa}$ cysteine-rich polypeptide belonging to a small family of secreted proteins characterized by a unique spacing of 10-11 cysteine residues, the resistin-like molecules ${ }^{[30,31]}$. In contrast to mouse resistin, human resistin is synthesized in cells other than adipocytes, predominantly in macrophages and monocytes particularly in the visceral adipose tissue characterized by a high metabolic turnover ${ }^{[31]}$. Elevated resistin levels caused by genetic or environmental factors such as obesity, inflammation and diet may play a pivotal role in the pathogenesis of insulin resistance, metabolic syndrome, t2DM, gestational diabetes, atherosclerosis, hypertension, cardiovascular disease and several malignancies such as breast, gastric, colorectal and esophageal cancers $^{[31,32]}$.

The majority of epidemiologic studies studying the association of serum resistin with $\mathrm{BC}$ have shown that 
hyperrestinemia in vivo is linked to the risk of $\mathrm{BC}$, particularly in postmenopausal women ${ }^{[4,33]}$. Our group has shown that mean serum resistin level was significantly higher in postmenopausal women suffering from BC than in age-matched control participants and women with benign breast lesions $(11.2 \pm 6.4$ vs $7.7 \pm 4.8$ vs 8.22 $\pm 6.1 \mathrm{ng} / \mathrm{mL}$ respectively, $P<0.05)$ both in univariate and multivariable analyses adjusting for age, date of diagnosis, education, family history of cancer, use of exogenous hormones, alcohol consumption, smoking status, physical activity, reproductive, metabolic, anthropometric, inflammatory markers and adipokines $(\mathrm{OR}=$ 1.17, $95 \%$ CI: $1.03-1.34, P=0.02)^{[4]}$.

In vitro findings have shown that resistin induced cancer cell proliferation through the PI3K/Akt signaling pathway ${ }^{[34]}$. A histopathological study has also indicated an association between tumor tissue resistin expression and malignant cancer behavior and prognosis in $\mathrm{BC}^{[35]}$. Serum resistin was reported as a good biomarker of malignant potential and stage progression in breast, esophageal, gastric, and colorectal adenocarcinoma, correlating positively with tumor size, cancer stage, histological grade and tumor markers ${ }^{[33]}$. In particular, circulating resistin levels may be an interesting biomarker for PBC reflecting advanced stage and inflammatory state ${ }^{[33]}$. Of interest, our group has shown that resistin level correlated with the tumor markers CA 15-3 and CEA, cancer stage, tumor size, histopathologic grade and lymph node invasion which are all associated with BC poor prognosis but not with anthropometric, metabolic parameters and hormone receptor status ${ }^{[30]}$. Although resistin's diagnostic performance was low based on receiver operating characteristic (ROC) curve analysis (0.72, 95\% CI: 0.64-0.79), it may constitute a BC biomarker reflecting advanced disease stage and inflammatory state ${ }^{[33]}$. Hence, resistin may represent a biomarker for $\mathrm{BC}$ development and progression, but may also act as a molecular mediator linking adipose tissue to breast carcinogenesis.

Possible mechanisms associating resistin with BC pathogenesis may involve: (1) Upregulation of proinflammatory cytokines via the $\mathrm{NF}-\kappa \mathrm{B}$ pathway, an important component of cancer-promoting machinery. Resistin may also promote a pro-thrombotic state via mediating the lipoprotein metabolism and inducing inflammation in a hypercoagulable environment observed in $\mathrm{BC}^{[32]}$; (2) Activation of signaling pathways playing an important role in inflammation and tumorigenesis. Resistin phosphorylated both MAPKs, such as Erk or p38, and Akt, a downstream substrate of PI3K, in several cell lines ${ }^{[36]}$; (3) Induction of the proangiogenic protein: vascular endothelial growth (VEGF) and formation of endothelial cell tubes contributing to metastasis; and (4) Induction of the expression of MMPs and reduction of MMPs tissue inhibitors participating in tumor invasiveness and metastasis ${ }^{[32]}$. Further mechanistic, larger prospective and longitudinal studies are required to confirm these findings and determine whether resistin may play a role as a BC tumor marker. More studies are needed to clarify resistin's ontological role in the association between obesity and BC.

\section{Nampt/visfatin and BC}

Visfatin, also known as nicotinamide phosphoribosyltransferase (Nampt) or pre-B cell colony-enhancing factor, constitutes a novel pleiotropic adipokine acting as an adipocytokine, a growth factor and an enzyme, found in the visceral fat, playing an important role in a variety of metabolic and stress responses as well as in the cellular energy metabolism, particularly nicotinamide adenine dinucleotide (NAD) biosynthesis ${ }^{[37]}$. Nampt plays a significant role in the enzymatic activity of an array of NADdependent enzymes which influence a variety of biological responses essential in cell survival and inflammation such as TNF- $\alpha$ biosynthesis ${ }^{[37]}$. Serum Nampt concentrations are elevated in obese women, obese children and adolescents, in patients with metabolic syndrome, t2DM, non-alcoholic fatty liver disease and coronary heart disease $^{[37-39]}$. So far, results have been conflicting regarding associations of Nampt with metabolic parameters ${ }^{[40]}$ underscoring the potential role of Nampt in the pathogenesis of low-grade chronic vascular inflammation in obesity and t2DM as a pro-inflammatory adipocytokine. Nampt was introduced as an insulin-mimetic molecule enhancing insulin signaling by binding insulin receptor at a different site than insulin but this was questioned later ${ }^{[37,41]}$. Increased Nampt expression has been shown in primary colorectal cancer and prostate cancer, malignancies that are related to overweight/obesity ${ }^{[37-39]}$. Interestingly, our group has reported that serum Nampt is significantly elevated in patients with $\mathrm{PBC}^{[39]}$, and may be a promising biomarker for $\mathrm{BC}^{[40]}$. Indeed, mean serum Nampt was significantly higher in PBC patients than in age-matched healthy controls and women with benign breast lesions $(57.9 \pm 31.2$ vs $43.6 \pm 28.1$ vs $42.9 \pm 18.1$ ng/mL respectively, $P<0.05)$. Postmenopausal women in the highest quartile of Nampt concentration $(>44.8$ $\mathrm{ng} / \mathrm{mL}$ ) present significantly higher risk for PBC adjusting for age, date of diagnosis, education, body mass index, waist circumference, years with menstruation, parity/age at first full term pregnancy, breastfeeding, family history of cancer, use of exogenous hormones, alcohol consumption, smoking status, homeostasis model assessment score, serum leptin and adiponectin concentrations $(\mathrm{OR}=7.93 \text {, 95\%CI: 2.52-24.9 })^{[39]}$. Moreover, in patients, Nampt was significantly associated with CA 15-3, hormone-receptor status, lymph node invasion but not with metabolic and anthropometric variables ${ }^{[40]}$. Circulating Nampt levels outperformed serum CA 15-3 only in discriminating between $\mathrm{PBC}$ cases with early cancer stage than those with late stage, and in differentiating particularly patients with ER-PR- breast malignancies ${ }^{[41]}$.

Elevated Nampt expression in BC tissues was reported to be associated with more malignant cancer behavior as well as adverse prognosis ${ }^{[42]}$. Pharmacologic inhibition of Nampt has been shown effective in a broad range of 
cancer cell lines and in mouse carcinoma models; however the definite role of Nampt in malignant diseases has yet to be elucidated ${ }^{[37-39]}$.

Pleiotropic Nampt may play a role in mammary epithelium tumorigenesis and provide an important link between obesity and PBC via the following mechanisms: (1) Nampt represents an essential enzyme in energy metabolism, circadian clock and cell longevity through intracellular NAD generation. NAD, a universal energyand signal-carrying molecule, is required in a plethora of intracellular processes such as redox reactions, DNA repair, G-protein coupled receptor signaling, intra-cellular calcium-mobilizing molecules, transcriptional regulation and activity of poly-ADP ribosyltransferases (PARPs) and sirtuins which modulate cell survival and cytokine responses ${ }^{[37,38]}$. In particular, sirtuins constitute a conserved family of NAD-dependent protein deacetylases and/or ADP-ribosyltransferases, being involved in longevity, metabolism, and stress and cytokine responses by deacetylating transcription regulators ${ }^{[43]}$. Seven sirtuins are expressed in mammals (SIRT 1-7), three of which (SIRT 3-5) are located in mitochondria ${ }^{[43]}$. Recent data has shown that SIRT1 overexpression suppresses apoptosis, promotes cell proliferation and angiogenesis, and contributes to the oncogenic potential of the ER $\alpha$ on estrogen-induced breast cancer growth ${ }^{[44]}$. On the contrary, SIRT3, a mitochondrial localized NAD-consuming tumor suppressor protein, may repress the Warburg effect on human breast cancer cell lines, a metabolic hallmark of many tumors characterized by a glycolytic switch even in the presence of oxygen providing cancer cells all the necessary substrates for biomass generation $^{[45]}$. Nampt activity prods cellular proliferation, shifts the balance toward cellular survival following a genotoxic insult and regulates the circadian clock machinery of some key transcriptions factors ${ }^{[37]}$. Further mechanistic studies using a metabolomics approach are needed in order to clarify the regulation of NAD biosynthesis and its temporal-spatial dynamics in $\mathrm{BC}$ cell metabolism. Therapeutic normalization of the NAD $+/ \mathrm{NADH}$ balance may inhibit metastasis and prevent BC progres$\operatorname{sion}^{[46]}$. Indeed, the combination of a Nampt small molecule inhibitor, FK866, with olaparib, a PARP inhibitor, inhibited triple-negative breast tumor growth in vivo to a greater extent than either single agent alone $\mathrm{e}^{[47]}$. A clear understanding of the interplay of NAD-consuming sirtuins and PARPs, Nampt and ER/PR at the molecular level may potentially open up new therapeutic avenues for BC treatment; (2) Nampt is a proliferative and antiapoptotic factor. Nampt stimulated the proliferation and DNA synthesis rate of MCF-7 human BC cells ${ }^{[37]}$. Nampt may play a role in $\mathrm{BC}$ development by enhancing the cell proliferation rate through stimulation of cell cycle progression. In prostate carcinogenesis, Nampt augmented PC3 cell proliferation by activating the MAPKs ERK-1/2 and p38 signaling pathways ${ }^{[48]}$. It is intriguing to identify the Nampt receptor and its signaling mechanism in BC pathogenesis; (3) Nampt may play a pro-an- giogenic, invasive and metastatic role. Increased Nampt concentrations were seen in PBC patients with advanced stage and worse prognosis ${ }^{[40]}$, and high Nampt expression in BC tissues was related to poor survival ${ }^{[42]}$. Nampt was shown to promote angiogenesis via activation of MAPK ERK-dependent pathway through endothelial fibroblast growth factor-2, and to enhance the VEGF factor via MAPK and PI3K/Akt signaling pathways ${ }^{[37,38]}$. By increasing the expression of MMP 2 and 9, and VEGF genes, Nampt may contribute to angiogenesis and metastasis in $\mathrm{BC}^{[37,38]}$; and (4) Nampt constitutes a pro-inflammatory adipocytokine linking obesity to PBC. Nampt stimulated several pro-inflammatory cytokines in human mononuclear cells and upregulated the production of IL-1 $\alpha$, IL-6, TNF- $\alpha$, intercellular adhesion molecule-1 and vascular cell adhesion molecule- 1 through the pro-inflammatory transcription factor $\mathrm{NF}-\kappa \mathrm{B}^{[37,38]}$. Therefore, Nampt may contribute to the pathogenesis of vascular inflammation linking obesity-a state of low grade inflammation- to PBC.

\section{FUTURE DIRECTIONS AND CLINICAL IM- PLICATIONS}

Obesity, its metabolic complications and $\mathrm{BC}$ have become major global health issues. Obesity increases the risk of $\mathrm{BC}$ incidence and mortality ${ }^{[7-9]}$. Imbalanced expression of adipokines could be involved in the association between obesity and BC, mainly postmenopausal. While understanding the connection of adipokines with $\mathrm{BC}$ might provide potential preventive and therapeutic strategies, lifestyle amelioration remains the most important component in preventing obesity-related PBC. Modulating adipokines might be a particularly attractive goal for BC prevention, specifically in overweight/obese women. A number of behavioral and drug interventions are associated with favorable modulation of main adipokines. Physical exercise, adoption of a balanced diet, weight reduction and bariatric surgery for morbidly obese women may augment plasma adiponectin and lower plasma leptin, resistin and Nampt concentrations reducing thus the risk of developing $\mathrm{BC}$. Pharmacologic agents such as metformin or PPAR- $\gamma$ agonists that increase adiponectin and decrease circulating resistin could be at the forefront of therapeutic modalities for $\mathrm{BC}^{[3]}$. Indeed, diabetic BC women using metformin experience a higher rate of pathological complete response to neoadjuvant chemotherapy than those taking other anti-diabetic treatments ${ }^{[49]}$. Lipid-lowering drugs such as statins and niacin, vitamin $\mathrm{C}$ and $\mathrm{D}$ supplementation, folic acid, oleic acid, calcium-channel blockers may also significantly modulate serum adipokines levels ${ }^{[3]}$. Some nutraceuticals such as curcumin have also been reported to decrease resistin and Nampt ${ }^{[5]}$.

Adiponectin use as a direct therapeutic drug in not available due to the difficulty in converting the full-size protein into a viable drug ${ }^{[3]}$. Nevertheless, ADP 355 , an adiponectinbased short peptide mimicking adiponectin's action sup- 
pressed in vivo the growth of orthotopic human breast cancer xenografts by $31 \%{ }^{[3]}$. AdipoR1/R2 agonists but also strategies to increase adiponectin receptors and upregulate adiponectin signaling pathway may provide novel therapeutic approaches for insulin resistance, $\mathrm{t} 2 \mathrm{DM}$ and $\mathrm{BC}^{[3]}$. Targeting the inhibition of adipokines that are elevated in $\mathrm{BC}$, either by antibody neutralization, antisense oligonucleotides or by antagonism of their receptor, could be an effective therapeutic strategy in BC, particularly in downregulating the tumor inflammatory microenvironment. In addition, if Nampt and resistin receptors, and their induced signaling pathways are clearly mapped out, inhibition of downstream targets may be further evaluated in BC therapeutics.

Recent data suggest that adipokines could be promising $\mathrm{BC}$ biomarkers in conjunction with other tumor markers, that reflect advanced stage, adverse prognosis and inflammatory state. Nevertheless, further studies are needed for the development of reliable and "user friendly" laboratory techniques (e.g., enzyme-linked immunosorbent assays) to assess adipokines, their isoforms and other adipokine-like molecules ${ }^{[3]}$, as well as their pathophysiologic relevance. There are still a number of unanswered practical questions in the clinical laboratory. What circulating levels of adipokines should be considered adnormal and what are their optimal levels for BC prevention? In the future, international standardization of levels and methodology procedures is also needed before full commercialization of adipokines as potential monitoring tools in BC.

Further evidence from mechanistic, larger prospective and longitudinal studies is required to determine exactly if and when adipokine concentrations are altered in $\mathrm{BC}$ and whether adpokines per se and/or other hormonal parameters connecting obesity with $\mathrm{BC}$ may be associated with $\mathrm{BC}$ etiopathogenesis. The hypothesis could also be tested by determining whether adipokines genetic polymorphisms are associated with $\mathrm{BC}$ prevalence. Moreover, the epigenetic regulation of the adipokine genes remains a Terra incognita.

In summary, there is evidence for a strong link between obesity-driven chronic inflammation, insulin resistance, adipokines and $\mathrm{BC}$. Advances in adipokine research may hold promise for the use of adipokines as potential prognostic markers and therapeutic targets. At the same time, several issues remain to be clarified in order to unmask the ontological role of some adipokines in BC pathophysiology. Reversing obesity-associated inflammation and dysfunction of the adipose tissue by lifestyle interventions such as weight reduction, physical activity and dietary modifications may present a clinically relevant contribution to decreasing BC risk or progression. Advances in the field of translational investigation may lead to tangible benefits to overweight/obese women who are at increased risk for BC.

\section{REFERENCES}

1 Renehan AG, Tyson M, Egger M, Heller RF, Zwahlen M.
Body-mass index and incidence of cancer: a systematic review and meta-analysis of prospective observational studies. Lancet 2008; 371: 569-578 [PMID: 18280327 DOI: 10.1016/ S0140-6736(08)60269-X]

2 Doyle SL, Donohoe CL, Lysaght J, Reynolds JV. Visceral obesity, metabolic syndrome, insulin resistance and cancer. Proc Nutr Soc 2012; 71: 181-189 [PMID: 22051112 DOI: 10.1017/S002966511100320X]

3 Dalamaga M, Diakopoulos KN, Mantzoros CS. The role of adiponectin in cancer: a review of current evidence. Endocr Rev 2012; 33: 547-594 [PMID: 22547160 DOI: 10.1210/ er.2011-1015]

4 Dalamaga M, Karmaniolas K, Papadavid E, Pelekanos N, Sotiropoulos G, Lekka A. Hyperresistinemia is associated with postmenopausal breast cancer. Menopause 2013; 20: 845-851 [PMID: 23481121]

5 Dalamaga M. Nicotinamide phosphoribosyl-transferase/visfatin: a missing link between overweight/obesity and postmenopausal breast cancer? Potential preventive and therapeutic perspectives and challenges. Med Hypotheses 2012; 79: 617-621 [PMID: 22922056 DOI: 10.1016/j.mehy.2012.07.036]

6 Wysocki PJ, Wierusz-Wysocka B. Obesity, hyperinsulinemia and breast cancer: novel targets and a novel role for metformin. Expert Rev Mol Diagn 2010; 10: 509-519 [PMID: 20465505 DOI: $10.1586 /$ erm.10.22]

7 Lorincz AM, Sukumar S. Molecular links between obesity and breast cancer. Endocr Relat Cancer 2006; 13: 279-292 [PMID: 16728564]

8 Macciò $\mathbf{A}$, Madeddu C. Obesity, inflammation, and postmenopausal breast cancer: therapeutic implications. ScientificWorldJournal 2011; 11: 2020-2036 [PMID: 22125453 DOI: 10.1100/2011/806787]

9 Pichard C, Plu-Bureau G, Neves-E Castro M, Gompel A. Insulin resistance, obesity and breast cancer risk. Maturitas 2008; 60: 19-30 [PMID: 18485631 DOI: 10.1016/ j.maturitas.2008.03.002]

10 Huang Z, Hankinson SE, Colditz GA, Stampfer MJ, Hunter DJ, Manson JE, Hennekens CH, Rosner B, Speizer FE, Willett WC. Dual effects of weight and weight gain on breast cancer risk. JAMA 1997; 278: 1407-1411 [PMID: 9355998]

11 Peacock SL, White E, Daling JR, Voigt LF, Malone KE. Relation between obesity and breast cancer in young women. Am J Epidemiol 1999; 149: 339-346 [PMID: 10025476]

12 Vona-Davis L, Rose DP. Type 2 diabetes and obesity metabolic interactions: common factors for breast cancer risk and novel approaches to prevention and therapy. Curr Diabetes Rev 2012; 8: 116-130 [PMID: 22268396]

13 Rose DP, Haffner SM, Baillargeon J. Adiposity, the metabolic syndrome, and breast cancer in African-American and white American women. Endocr Rev 2007; 28: 763-777 [PMID: 17981890]

14 Alberti KG, Zimmet P, Shaw J. The metabolic syndrome--a new worldwide definition. Lancet 2005; 366: 1059-1062 [PMID: 16182882]

15 Duan XF, Tang P, Li Q, Yu ZT. Obesity, adipokines and hepatocellular carcinoma. Int J Cancer 2013; 133: 1776-1783 [PMID: 23404222]

16 Moon HS, Dalamaga M, Kim SY, Polyzos SA, Hamnvik OP, Magkos F, Paruthi J, Mantzoros CS. Leptin's role in lipodystrophic and nonlipodystrophic insulin-resistant and diabetic individuals. Endocr Rev 2013; 34: 377-412 [PMID: 23475416 DOI: 10.1210/er.2012-1053]

17 Dalamaga M, Chou SH, Shields K, Papageorgiou P, Polyzos SA, Mantzoros CS. Leptin at the intersection of neuroendocrinology and metabolism: current evidence and therapeutic perspectives. Cell Metab 2013; 18: 29-42 [PMID: 23770129 DOI: 10.1016/j.cmet.2013.05.010]

18 Catalano S, Mauro L, Bonofiglio D, Pellegrino M, Qi H, Rizza P, Vizza D, Bossi G, Andò S. In vivo and in vitro evidence that PPARY ligands are antagonists of leptin signaling 
in breast cancer. Am J Pathol 2011; 179: 1030-1040 [PMID: 21704006 DOI: 10.1016/j.ajpath.2011.04.026]

19 Ray A. Adipokine leptin in obesity-related pathology of breast cancer. J Biosci 2012; 37: 289-294 [PMID: 22581334]

20 Grossmann ME, Cleary MP. The balance between leptin and adiponectin in the control of carcinogenesis - focus on mammary tumorigenesis. Biochimie 2012; 94: 2164-2171 [PMID: 22728769 DOI: 10.1016/j.biochi.2012.06.013]

21 Ray A, Cleary MP. Obesity and breast cancer: a clinical biochemistry perspective. Clin Biochem 2012; 45: 189-197 [PMID: 22178111 DOI: 10.1016/j.clinbiochem.2011.11.016]

22 Ollberding NJ, Kim Y, Shvetsov YB, Wilkens LR, Franke AA, Cooney RV, Maskarinec G, Hernandez BY, Henderson BE, Le Marchand L, Kolonel LN, Goodman MT. Prediagnostic leptin, adiponectin, C-reactive protein, and the risk of postmenopausal breast cancer. Cancer Prev Res (Phila) 2013; 6: 188-195 [PMID: 23466816 DOI: 10.1158/1940-6207. CAPR-12-0374]

23 Mantzoros C, Petridou E, Dessypris N, Chavelas C, Dalamaga M, Alexe DM, Papadiamantis Y, Markopoulos C, Spanos E, Chrousos G, Trichopoulos D. Adiponectin and breast cancer risk. J Clin Endocrinol Metab 2004; 89: 1102-1107 [PMID: 15001594]

24 Ziemke F, Mantzoros CS. Adiponectin in insulin resistance: lessons from translational research. Am J Clin Nutr 2010; 91: 258S-261S [PMID: 19906806 DOI: 10.3945/ajcn.2009.28449C]

25 Fabian CJ. Adiponectin: a risk biomarker and attractive target for chemoprevention. J Clin Oncol 2012; 30: 124-126 [PMID: 22162567 DOI: 10.1200/JCO.2011.38.5500]

26 Miyoshi Y, Funahashi T, Kihara S, Taguchi T, Tamaki Y, Matsuzawa Y, Noguchi S. Association of serum adiponectin levels with breast cancer risk. Clin Cancer Res 2003; 9: 5699-5704 [PMID: 14654554]

27 Macis D, Gandini S, Guerrieri-Gonzaga A, Johansson H, Magni P, Ruscica M, Lazzeroni M, Serrano D, Cazzaniga M, Mora S, Feroce I, Pizzamiglio M, Sandri MT, Gulisano M, Bonanni B, Decensi A. Prognostic effect of circulating adiponectin in a randomized $2 \times 2$ trial of low-dose tamoxifen and fenretinide in premenopausal women at risk for breast cancer. J Clin Oncol 2012; 30: 151-157 [PMID: 22162577 DOI: 10.1200/JCO.2011.35.2237]

28 Körner A, Pazaitou-Panayiotou K, Kelesidis T, Kelesidis I, Williams CJ, Kaprara A, Bullen J, Neuwirth A, Tseleni S, Mitsiades N, Kiess W, Mantzoros CS. Total and high-molecular-weight adiponectin in breast cancer: in vitro and in vivo studies. J Clin Endocrinol Metab 2007; 92: 1041-1048 [PMID: 17192291]

29 Delort L, Jardé T, Dubois V, Vasson MP, Caldefie-Chézet F. New insights into anticarcinogenic properties of adiponectin: a potential therapeutic approach in breast cancer? Vitam Horm 2012; 90: 397-417 [PMID: 23017724 DOI: 10.1016/B9780-12-398313-8.00015-4]

30 Steppan CM, Bailey ST, Bhat S, Brown EJ, Banerjee RR, Wright CM, Patel HR, Ahima RS, Lazar MA. The hormone resistin links obesity to diabetes. Nature 2001; 409: 307-312 [PMID: 11201732]

31 Schwartz DR, Lazar MA. Human resistin: found in translation from mouse to man. Trends Endocrinol Metab 2011; 22: 259-265 [PMID: 21497511 DOI: 10.1016/j.tem.2011.03.005]

32 Filková M, Haluzík M, Gay S, Senolt L. The role of resistin as a regulator of inflammation: Implications for various human pathologies. Clin Immunol 2009; 133: 157-170 [PMID: 19740705 DOI: 10.1016/j.clim.2009.07.013]

33 Dalamaga M, Sotiropoulos G, Karmaniolas K, Pelekanos N, Papadavid E, Lekka A. Serum resistin: a biomarker of breast cancer in postmenopausal women? Association with clinicopathological characteristics, tumor markers, inflammatory and metabolic parameters. Clin Biochem 2013; 46: 584-590 [PMID: 23321342 DOI: 10.1016/j.clinbiochem.2013.01.001]

34 Kim HJ, Lee YS, Won EH, Chang IH, Kim TH, Park ES, Kim
MK, Kim W, Myung SC. Expression of resistin in the prostate and its stimulatory effect on prostate cancer cell proliferation. BJU Int 2011; 108: E77-E83 [PMID: 21050358 DOI: 10.1111/j.1464-410X.2010.09813.x]

35 Lee YC, Chen YJ, Wu CC, Lo S, Hou MF, Yuan SS. Resistin expression in breast cancer tissue as a marker of prognosis and hormone therapy stratification. Gynecol Oncol 2012; 125: 742-750 [PMID: 22370603 DOI: 10.1016/j.ygyno.2012.02.032]

36 Calabro P, Samudio I, Willerson JT, Yeh ET. Resistin promotes smooth muscle cell proliferation through activation of extracellular signal-regulated kinase $1 / 2$ and phosphatidylinositol 3-kinase pathways. Circulation 2004; 110: 3335-3340 [PMID: 15545519]

37 Garten A, Petzold S, Körner A, Imai S, Kiess W. Nampt: linking NAD biology, metabolism and cancer. Trends Endocrinol Metab 2009; 20: 130-138 [PMID: 19109034 DOI: 10.1016/ j.tem.2008.10.004]

38 Zhang LQ, Heruth DP, Ye SQ. Nicotinamide Phosphoribosyltransferase in Human Diseases. J Bioanal Biomed 2011; 3: 13-25 [PMID: 22140607]

39 Dalamaga M, Karmaniolas K, Papadavid E, Pelekanos N, Sotiropoulos G, Lekka A. Elevated serum visfatin/nicotinamide phosphoribosyl-transferase levels are associated with risk of postmenopausal breast cancer independently from adiponectin, leptin, and anthropometric and metabolic parameters. Menopause 2011; 18: 1198-1204 [PMID: 21712732 DOI: 10.1097/gme.0b013e31821e21f5]

40 Dalamaga M, Archondakis S, Sotiropoulos G, Karmaniolas K, Pelekanos N, Papadavid E, Lekka A. Could serum visfatin be a potential biomarker for postmenopausal breast cancer? Maturitas 2012; 71: 301-308 [PMID: 22261365 DOI: 10.1016/ j.maturitas.2011.12.013]

41 Fukuhara A, Matsuda M, Nishizawa M, Segawa K, Tanaka M, Kishimoto K, Matsuki Y, Murakami M, Ichisaka T, Murakami $\mathrm{H}$, Watanabe E, Takagi T, Akiyoshi M, Ohtsubo T, Kihara S, Yamashita S, Makishima M, Funahashi T, Yamanaka S, Hiramatsu R, Matsuzawa Y, Shimomura I. Visfatin: a protein secreted by visceral fat that mimics the effects of insulin. Science 2005; 307: 426-430 [PMID: 15604363]

42 Lee YC, Yang YH, Su JH, Chang HL, Hou MF, Yuan SS. High visfatin expression in breast cancer tissue is associated with poor survival. Cancer Epidemiol Biomarkers Prev 2011; 20: 1892-1901 [PMID: 21784959 DOI: 10.1158/1055-9965. EPI-11-0399]

43 Finkel T, Deng CX, Mostoslavsky R. Recent progress in the biology and physiology of sirtuins. Nature 2009; 460: 587-591 [PMID: 19641587 DOI: 10.1038/nature08197]

44 Elangovan S, Ramachandran S, Venkatesan N, Ananth S, Gnana-Prakasam JP, Martin PM, Browning DD, Schoenlein PV, Prasad PD, Ganapathy V, Thangaraju M. SIRT1 is essential for oncogenic signaling by estrogen/estrogen receptor a in breast cancer. Cancer Res 2011; 71: 6654-6664 [PMID: 21920899 DOI: 10.1158/0008-5472.CAN-11-1446]

45 Finley LW, Carracedo A, Lee J, Souza A, Egia A, Zhang J, Teruya-Feldstein J, Moreira PI, Cardoso SM, Clish CB, Pandolfi PP, Haigis MC. SIRT3 opposes reprogramming of cancer cell metabolism through HIF1a destabilization. Cancer Cell 2011; 19: 416-428 [PMID: 21397863 DOI: 10.1016/ j.ccr.2011.02.014]

46 Santidrian AF, Matsuno-Yagi A, Ritland M, Seo BB, LeBoeuf SE, Gay LJ, Yagi T, Felding-Habermann B. Mitochondrial complex I activity and NAD + / NADH balance regulate breast cancer progression. J Clin Invest 2013; 123: 1068-1081 [PMID: 23426180 DOI: 10.1172/JCI64264]

47 Bajrami I, Kigozi A, Van Weverwijk A, Brough R, Frankum J, Lord CJ, Ashworth A. Synthetic lethality of PARP and NAMPT inhibition in triple-negative breast cancer cells. EMBO Mol Med 2012; 4: 1087-1096 [PMID: 22933245 DOI: 10.1002/emmm.201201250]

48 Patel ST, Mistry T, Brown JE, Digby JE, Adya R, Desai KM, 
Randeva HS. A novel role for the adipokine visfatin/pre-B cell colony-enhancing factor 1 in prostate carcinogenesis. Peptides 2010; 31: 51-57 [PMID: 19819277 DOI: 10.1016/ j.peptides.2009.10.001]

49 Duggan C, Irwin ML, Xiao L, Henderson KD, Smith AW,
Baumgartner RN, Baumgartner KB, Bernstein L, Ballard-Barbash R, McTiernan A. Associations of insulin resistance and adiponectin with mortality in women with breast cancer. $J$ Clin Oncol 2011; 29: 32-39 [PMID: 21115858 DOI: 10.1200/ JCO.2009.26.4473]

P-Reviewer Jain A S- Editor Wen LL L- Editor A E- Editor Liu XM

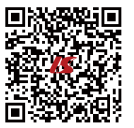




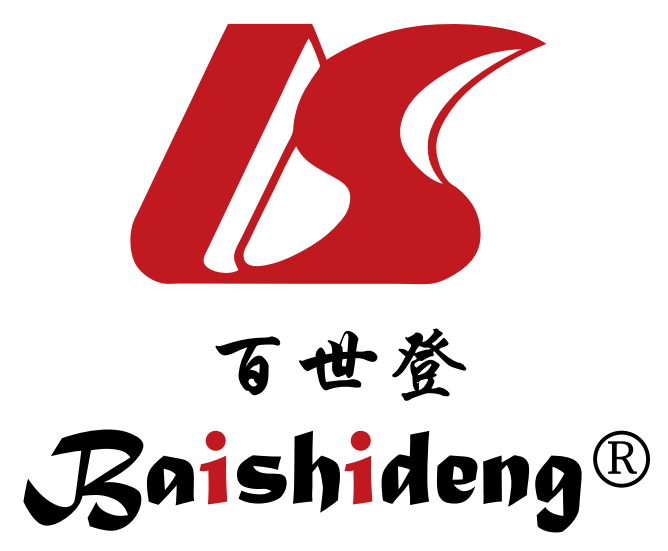

Published by Baishideng Publishing Group Co., Limited Flat C, 23/F., Lucky Plaza, 315-321 Lockhart Road, Wan Chai, Hong Kong, China

Fax: $+852-65557188$

Telephone: $+852-31779906$

E-mail: bpgoffice@wjgnet.com

http://www.wjgnet.com

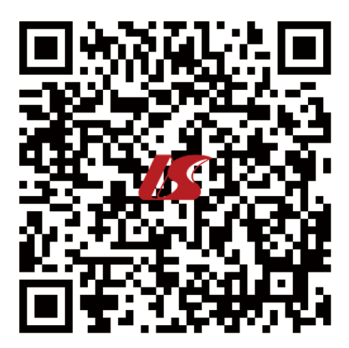

